\title{
Single Layer Perceptrons Neural Networks for Online Eigenstructure Assignment in MIMO Systems
}

\author{
Bruno F. Coelho* Daniel T. Simões ${ }^{* *}$ João Viana da F. Neto ${ }^{* * *}$ \\ Patrícia Helena M. Rêgo ${ }^{* * * *}$ \\ * Federal University of Maranhão \\ MA,Brazil (e-mail: brunofranca08@gmail.com). \\ ** Federal University of Maranhão \\ MA,Brazil (e-mail: eng.daniel.trovao@gmail.com). \\ *** Federal University of Maranhão \\ MA,Brazil (e-mail: jviana@dee.ufma.br). \\ **** State University of Maranhão \\ MA,Brazil(e-mail: phmrego@yahoo.com.br).
}

\begin{abstract}
To improve the stability performance and the response form of MIMO systems, the control of the modes is relevant to impose the design specifications. A bioinspired method for online eigenstructure assignment for the design of multivariable control systems is presented in this paper. The method is based on artificial neural networks for the execution of the control law and for training the controller gains by state feedback. Aiming at online tuning of state space controllers, the gain matrix that satisfies all the design specifications, a proposal is presented to compute the gain matrices that best meet a given operating range of MIMO dynamic systems. The proposal is evaluated in a mathematical model that represents a fourth order RLC circuit with two input voltages and two controllable voltage levels.
\end{abstract}

Keywords: Eigenstructure Assignment, Online, Neural Network.

\section{INTRODUCTION}

Bio-inspired approaches, such as neural networks and genetic algorithms associated with optimization structures, are approaches for the formulation and development of methods for solving complete and partial eigenstructure assignment problems in multiple-input multiple-output (MIMO) systems. The complete assignment takes into account the specification of all the eigenvalues and the components of the associated eigenvectors, while partial assignment considers only the specification of a few eigenstructure pairs and elements of the eigenvectors.

A relevant application of eigenstructure assignment (EA) can be found in (Gan and Danai, 1999), where a model-based recurrent neural network (MBRNN) is explored in fault diagnosis. The main feature of this network is the incorporation of analytical knowledge of the system into its structure, which reduces the demand for training. In (Liu and $\mathrm{Lu}, 1997$ ), the authors develop a synthesis approach for associative memories with learning and forgetting abilities through feedback artificial neural networks. The proposed synthesis technique uses the EA method. The generated networks are able to learn new patterns as well as forget the learned patterns without the need to recalculate all interconnection weights and external inputs. In many aspects, the results are significant improvements over the external product method, the projection learning rule, and the pseudo-inverse method with stability constraints.

The eigenstructure assignment problem is of fundamental importance in control theory and applications, since the stability and dynamic behavior of a linear multivariable system are governed by the eigenstructure of the system ( $\mathrm{Lu}$ et al., 1993). In general, the response speed is determined by the assigned eigenvalues, while the response shape is provided by the assigned eigenvectors. The eigenstructure assignment is an excellent method for incorporating classical specifications on damping, settling time and mode decoupling into a MIMO structure (Sobel and Lallman, 1988) and has shown to be a useful tool for the flight control design (Sobel et al., 1994). The EA technique is used to design flight control laws for aircraft with many control effects, and the technique together with the appropriate feedforward design can achieve static decoupling with internal stability, which is an important requirement in many flight control systems.

As shown in (Albostan and Gökasan, 2018), the physical modes of a system can be decoupled by adequate modeling of eigenvectors. That presents the design of a robust controller based on the eigenstructure assignment technique. While maintaining the desired eigenvector structure as much as possible, the NelderMead optimization algorithm is implemented to search for the optimal robust solution. Different optimization cost functions are investigated in terms of eigenvalue sensitivity and stability robustness. The effectiveness of the control system is demonstrated with nonlinear simulations on the F-16 aircraft mathematical model.

Another interesting application of eigenstructure assignment is proposed in (Konara and Annakkage, 2016). There, the design of a power system damping controller using partial right EA is presented. The EA technique selects a set of closedloop eigenvalues along with its right or left eigenvectors. The 
selection of eigenvectors offers extra flexibility that is explored when designing a robust damping controller that provides the required damping under multiple operating conditions. Unlike the algorithms previously proposed in the literature, such an assignment incorporates different operating conditions into the design stage itself to obtain a robust damping controller, and most importantly, when the measured signals are remotely used in the controller, the robustness can be improved.

The aim of the present paper is to develop a method for eigenstructure assignment in MIMO systems by state feedback as well as to develop an algorithm for eigenstructure assignment that is based on Single Layer Perceptrons Artificial Neural Network (SLP-ANN) for execution of the state feedback control law and a Structured Artificial Neural Network (S-ANN) for SLP-ANN training. The proposed method is compared with the classical methods which are presented in (Liu and Patton, 1998).

This work is organized in sections as follows: Section 2 provides the preliminaries, addressing the classical formulation of the EA problem and solution methods. Section 3 is devoted to the formulation and the proposed solutions to solve the EA problem in a bio-inspired and online manner. Section 4 describes the algorithm that composes the control system for the eigenstructure assignment based on neural networks, such as feedforward ANN and structured ANN. Section 5 shows the results from an evaluation in a mathematical model that represents a fourth order RLC circuit. Finally, Section 6 presents conclusions and comments about the development of the work and perspectives of implementation in real world systems.

\section{PROBLEM FORMULATION OF EA}

\subsection{System Model}

The state-space description of the dynamic system is given by

$$
\begin{aligned}
& \dot{x}=A x+B u, \\
& y=C x+D u,
\end{aligned}
$$

where $A \in \mathfrak{R}^{n \times n}$ is the matrix that represents the dynamics of the system, $B \in \mathfrak{R}^{n \times q}$ is the control matrix, $C \in \mathfrak{R}^{p \times n}$ is the output matrix, $D \in \mathfrak{R}^{p \times q}$ is the direct transmission matrix, $x \in \mathfrak{R}^{n}$ is the state vector, and $u \in \mathfrak{R}^{q}$ is the control input vector.

\subsection{Problem Statement}

The problem to be solved is to determine the state feedback gain matrix $K$ that assigns the right eigenstructure specified by the designer, i.e., a given set of eigenvalues $\left\{\lambda_{d i}\right\}$ and right eigenvectors $\left\{V_{d i}\right\}$. The control law that imposes the desired eigenstructure is set as

$$
u=-K x,
$$

where $K \in \mathfrak{R}^{q \times n}$ is state feedback gain matrix. That law is applied to the MIMO dynamic system of Eqs.(1) and (2). The closed-loop system is given by

$$
\dot{x}=A_{c l} x,
$$

where $A_{c l}$ is the closed-loop matrix that is given by

$$
A_{c l}=(A-B K)
$$

The desired eigenstructure assignment is represented by the set $\left\{\Lambda_{d}, V_{d}\right\}$, where $\Lambda_{d}$ is the eigenvalue diagonal matrix of the closed-loop dynamic system defined as

$$
\Lambda_{d}=\operatorname{diag}\left[\begin{array}{llll}
\lambda_{d 1} & \lambda_{d 2} & \cdots & \lambda_{d n}
\end{array}\right],
$$

and $V_{d}$ is the associated eigenvector matrix which is given by

$$
V_{d}=\left[\begin{array}{llll}
v_{d 1} & v_{d 2} & \cdots & v_{d n}
\end{array}\right],
$$

where $v_{d i} \in \mathbb{C}^{n}$ and $\lambda_{d i} \in \mathbb{C}$.

In order to ensure the desired EA, the gain matrix $K$ must satisfy

$$
A_{c l} V_{d}=\left[\begin{array}{lllll}
\lambda_{d 1} v_{d 1} & \lambda_{d 2} v_{d 2} & \cdots & \lambda_{d n} v_{d n}
\end{array}\right]
$$

From (8), one notes that the problem is to determine the solution of a set of $n$ linear systems. In this way, for each pair $\left(\lambda_{i}, v_{i}\right)$, one has a linear system to be solved. In the case of complete state feedback, the $n$ linear systems must be solved, which does not occur in the case of partial eigenstructure assignment. Consequently, the computational complexity of the iterative process to determine the gain vector $K$ depends on the number of control variables and the order $n$ of the system, which are related to the size of gain matrix and the number of linear systems.

Replacing (5) in (8), considering that $n$ linear systems in terms of the components of $v_{i}$ and eigenvalues $\lambda_{i}$ must be solved for the right eigenstructure assignment problem, one has

$$
(A-B K) v_{i}=\lambda_{i} v_{i}
$$

Thus, the $A E$ problem in terms of the $i$-th calculated eigenstructure is given by

$$
A v_{i}-B K v_{i}=\lambda_{d} v_{i}
$$

Writing (10) explicitly in terms of gains $K$, i.e., the closedloop state feedback gain matrix is unknown, the eigenstructure assignment problem can be described as a null-space problem. The classic form that relates the assignment eigenstructure in its general form is given by

$$
\left(A-\lambda_{i} I\right) v_{i}-B K v_{i}=0
$$

(11), which is nonlinear for the unknowns $K$ and $v_{i}$, in its vector form is expressed by

$$
\left[\begin{array}{ll}
\left(A-\lambda_{i} I\right) & B
\end{array}\right]\left[\begin{array}{c}
v_{i} \\
-K v_{i}
\end{array}\right]=0, \quad i=1, \ldots, n
$$

In order to solve the EA problem, the following mathematical artifice is used, taking into account that the eigenstructure is specified. So, the auxiliary variable $w_{i}$ is inserted into (12) and is given by

$$
w_{i}=K v_{i}
$$

Thus, the value of $w_{i}$ for a given $v_{i}$ is calculated in the search context of a given gain matrix $K$ that must satisfy the set of desired eigenstructure constraints, i.e., $\left\{\Lambda_{d}, V_{d}\right\}$. 
Replacing (13) in (12), one obtains an equation that relates the pair $\left(v_{i}, \lambda_{i}\right)$ with the auxiliary variable $W$. The new relation is given by

$$
\left(A-\lambda_{i} I\right) v_{i}-B w_{i}=0
$$

The direct solution is given by the inverse of the Matrix $(A-$ $\left.\lambda_{i} I\right)$. Hence, the gain matrix $W$, according to (13), is given by

$$
v_{i}=\left(A-\lambda_{i} I\right)^{-1} B w_{i}
$$

From (15) can be rearranged to

$$
w_{i}=\left(B^{T} B\right)^{-1} B^{T}(A-\lambda I) v_{i}
$$

Once $w_{i}$ is calculated from (16), the feedback gain $K$ becomes

$$
K=W V_{d}^{-1}
$$

The use of the neural network is justified because the studied systems can be overdetermined or underdetermined in largescale, ill-conditioned, and systems that have associated uncertainties. Also, such an ANN structure avoids the computation of matrix inverse.

\subsection{ANN Architecture for EA}

In addition to ANN for the control $u=K x$, the SLP-ANN training is performed by S-ANN. The inputs of the network are the states of the system and its outputs are the control laws, the input-output pair is used to generate the system error.

The gain matrix $K$ expressed in terms of its elements is given by

where $K \in \mathfrak{R}^{q \times n}$.

$$
K=\left[K_{i j}\right], \quad i=1, \ldots, q, \quad j=1, \ldots, n
$$

The control inputs are represented in terms of scalar equations as follows

$$
u_{i}=-\left\langle K_{i}, x\right\rangle, \quad i=1, \ldots q
$$

where $K_{i}=\left[\begin{array}{llll}K_{i 1} & K_{i 2} & \ldots & K_{i n}\end{array}\right]^{T}$.

Fig. 1 shows the general configuration of a closed-loop state space system with an SLP-ANN This network will provide online an $K$ state feedback matrix capable of assign the eigenstructure desired. The training algorithm depends on the parametric estimation of the matrices that represent the dynamics of the system and also the eigenstructure desired.

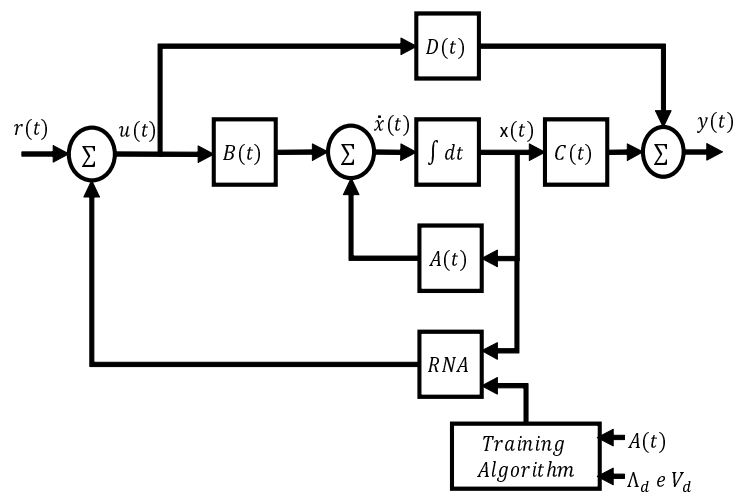

Figure 1. Diagram of system blocks in state space, in closed loop with neural network.
The neural network architecture that represents the online and bioinspired control for the eigenstructure assignment by state feedback. From this equation, a block diagram of the SLP-ANN architecture is constructed, which is shown in Fig. 2.

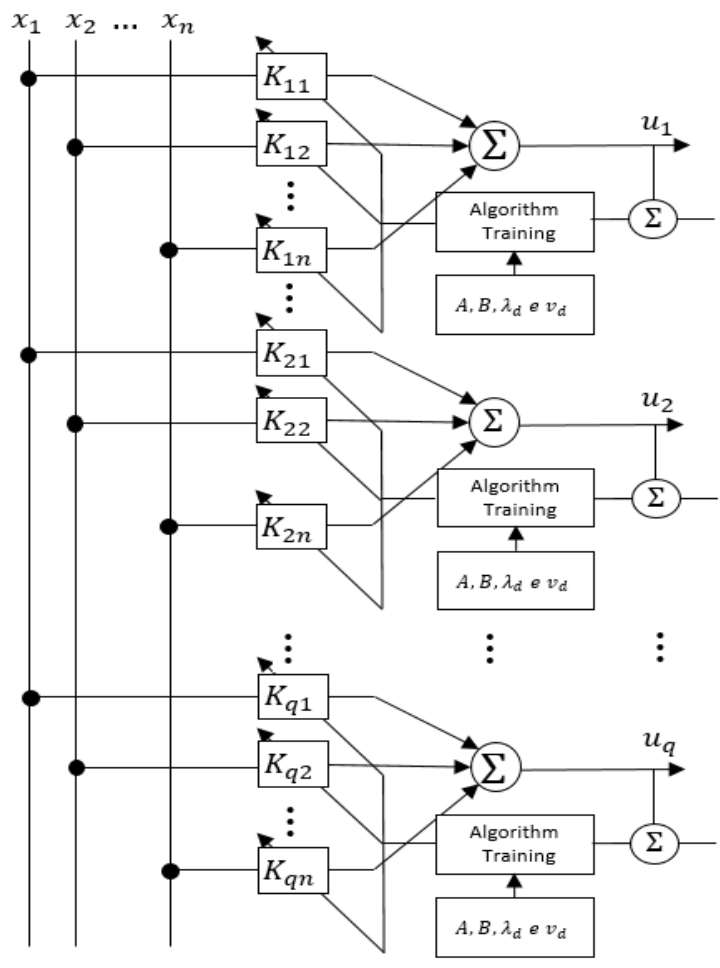

Figure 2. SLP-ANN architecture of the control device for online eigenstructure assignment.

The SLP-ANN architecture of the control device of Fig. 2 is an abstraction for the development of an embedded system for online eigenstructure assignment based on bio-inspired learning for SLP-ANN training. In this network, the inputs are the states sampled for time intervals $T_{S}$ (Gajski et al., 2009). Furthermore, it is observed that the SLP-ANN output is compared to the specified control input $U_{d i}$. This input is assembled from each pair $\left(V_{d i}, \lambda_{d i}\right)$ of the desired eigenstructure. The network training algorithm receives the result of the comparison between the actual value and the desired value. Thus, the rule for adjusting the network synaptic weights is based on the difference between these two values. Considering that the output signal for a given value of the activation function representing the post-synaptic activity and the resulting signal of the linear combiner which is called the pre-synaptic signal, according to the ANN theory that is supported by the Hebb postulates (Haykin, 1999).

\section{SUPERVISED TRAINING OF CONTROL DEVICE}

The training of the neural network which performs the state feedback control structure is based on the structured neural network approach (S-ANN). The structured neural network consists of a projection network and a network for implementing the gradient projection algorithm, where the projection network is constructed from specially structured linear neurons with a special training algorithm (Wang and Wan, 2001). The S-ANN solution must solve $n$ linear systems of the type

$$
A_{c l} v_{i}=\lambda_{i} v_{i}
$$


(19) in terms of a null space problem is given by

$$
\left[\begin{array}{ll}
(A-I \lambda) & -B
\end{array}\right]\left[\begin{array}{c}
v_{i} \\
w_{i}
\end{array}\right]=0
$$

where $W$ is the intermediate vector that allows to linearize the system of equations of the structure of (19).

Taking into account that the $i$-th desired eigenstructure is represented by the pair $\left(v_{d i}, \lambda_{d i}\right)$, one can rewrite the equation of desired $A E$ in terms of $w_{i}$ as follows

$$
\left(A-\lambda_{d i} I\right) v_{d i}-B w_{i}=0 .
$$

In the structured neural network, the transformed inputs are given by $w_{i}$ and the outputs are given by the transformation of the pairs $\left(v_{d i}, \lambda_{d i}\right)$.

According to (21), the iterative process for network training of the control device is henceforth referred to as an actor in the sense that it executes the decision based on training of the feedforward-type neural network via S-ANN. One of the most important equations for determining the state feedback matrix is set as

$$
B w_{i}=\left(A-\lambda_{d i} I\right) v_{d i},
$$

where $B \in \mathfrak{R}^{n \times q}$ and $w_{i} \in \mathfrak{R}^{q \times 1}$.

The architecture of this training network, proposed from (22), in terms of its coefficients and in its matrix form is given by

$$
\left[\begin{array}{cccc}
b_{11} & b_{12} & b_{13} & b_{1 n} \\
b_{21} & b_{22} & \cdots & b_{2 n} \\
\vdots & \vdots & \ddots & \vdots \\
b_{n 1} & b_{n 2} & \cdots & b_{n q}
\end{array}\right]\left[\begin{array}{c}
\bar{W}_{11} \\
\bar{W}_{21} \\
\vdots \\
\bar{W}_{q 1}
\end{array}\right]=\left[\begin{array}{c}
\bar{a}_{11} \\
\bar{a}_{21} \\
\vdots \\
\bar{a}_{2 n}
\end{array}\right]
$$

where $\bar{a}$ is the vector of singularities of the pair $\left(v_{d i}, \lambda_{d i}\right)$.

In the compact form, the system of linear equations can be rewritten by

$$
B \bar{W}=\bar{a},
$$

where $\bar{a}=\left(A-\lambda_{d i} I\right) v_{d i}$.

For the purpose of solving the null space problem, (20) is represented in the form of a linear system which is given by

$$
\left(A-I \lambda_{d}\right) V_{d}=B W
$$

where

$$
W=K V_{d}
$$

From (24), which represents the network for training the synaptic weights of the control device, the architecture is constructed and the proposal of an algorithm for training of this network is presented. The structured network has as input the singularity vector of the pair $\left(V_{d i}, \lambda_{i}\right)$ and as output the control vector $K$.

The structured ANN learning rule is based on the gradient method, specifically, the steepest descent method. The learning rule for this network is given by the variation of $\Delta W$ according to (Haykin, 1999), i.e.

$$
\frac{d K(t)}{d t}=-\mu \nabla_{K} \varepsilon(x)
$$

where $\mu$ is the training rate that belongs to the matrix space $\mu \in \mathfrak{R}^{q \times n}$, with $\mu$ definite positive. According to Eqs. (27) and (23), gradient for the problem, is given by

$$
\frac{d K(t)}{d t}=-\mu B\left(B W-\bar{a}_{i}\right)
$$

In this formulation, the error according to the equations (24)(28), in terms of the system parameters and their transformations, is expressed as

$$
\frac{d K(t)}{d t}=-\mu B^{T} e
$$

where $e$ is the error that is given by

$$
e=B W-\bar{a}_{i}
$$

and the initial conditions are given by $K(0)=K_{0}$.

It is noteworthy to point out some relevant comments on the properties of the proposed neural network, according to the number of inputs and outputs of the dynamic system: a) Considering the system of (24), the error model is suitable to solve the problem of linear systems, taking into account that the equations may be of the determined $(m=n)$, over-determined $(m>n)$ or sub-determined $(m<n)$ types; b) ANN solves problems where the $B$ matrix has incomplete rank, and it also solves situations where the matrix $A$ is ill-conditioned.

The determination of the gain $K$ is performed from (31) which depends on the calculation of $W$. Using the LS method requires the calculation of the pseudo-inverse, which due to problems of ill conditioning makes the process unreliable and may present no solution. To bypass this problem, $K$ is represented as linear systems to the right. Applying the conjugate transpose in $W$, the problem formulation to determine the gain $K$ is given by

$$
V_{d}^{T} K^{T}=W^{T}
$$

\section{SLP-ANN ALGORITHM}

The EA-SLP-ANN algorithm for eigenstructure assignment is assembled of functional blocks. These blocks are developed according to embedded system approaches (Gajski et al., 2009) (Lee and Seshia, 2016).

$$
\begin{array}{ll}
\text { ALGORITHM }-E A-S L P-A N N \\
1 \\
2 \text { DSetup (Initial Conditions) } \\
3 \text { Model_Sistem_Dynamic } \\
4 \text { Proced_Especific_Autoestrutura } \\
4 E A-A N N-\text { Core }
\end{array}
$$

The $E A-A N N-$ Core algorithm, step 4 of the $E A-S L P-$ $A N N$ algorithm, of the structured neural network for the determination of gains consists of two S-ANNs. One of the networks determines the vector $W$ and the other determines the state feedback gain $K$. 
Algorithm EA-ANN-Core

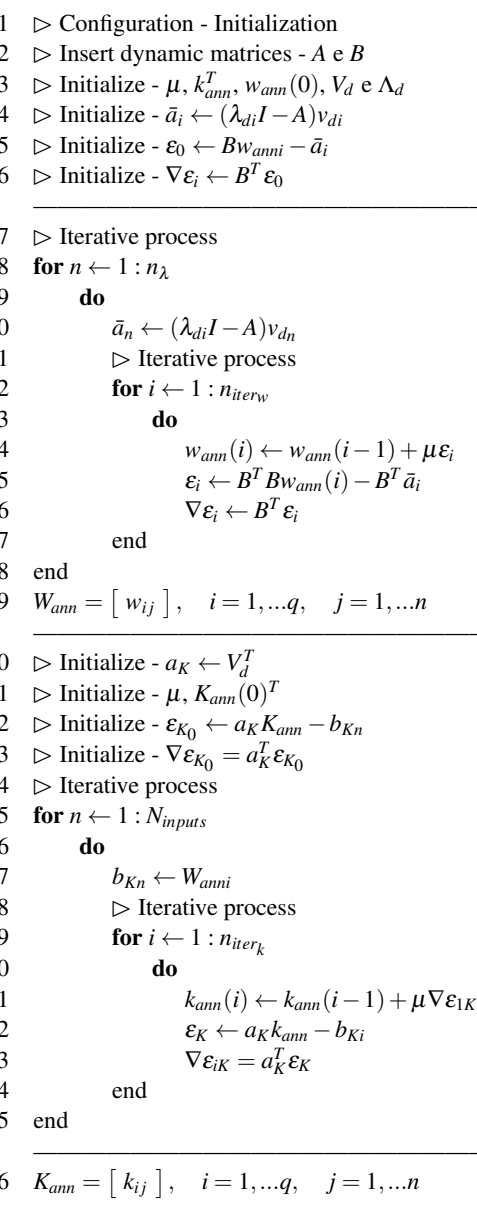

\section{COMPUTATIONAL EXPERIMENTS}

In order to investigate and verify the proposed neural network model for solving the eigenstructure assignment problem, computational simulations were performed using the weight updating formula. The performance of SLP-ANN is evaluated in a model of RLC electric circuit plant, as shown

$$
\begin{gathered}
A=\left[\begin{array}{cccc}
-1 / \mathrm{C}_{\text {cap }} \mathrm{R}_{1} & 0 & 1 / \mathrm{C}_{\text {cap }} & 0 \\
0 & -1 / \mathrm{C}_{\text {cap }} \mathrm{R}_{1} & 0 & -1 / \mathrm{C}_{\text {cap }} \\
-1 / \mathrm{L}_{\text {ind }} & 0 & -\mathrm{R}_{2} / \mathrm{L}_{\text {ind }} & -\mathrm{R}_{2} / \mathrm{L}_{\text {ind }} \\
0 & 1 / \mathrm{L}_{\text {ind }} & -\mathrm{R}_{2} / \mathrm{L}_{\text {ind }} & -\mathrm{R}_{2} / \mathrm{L}_{\text {ind }}
\end{array}\right], \\
B=\left[\begin{array}{cc}
0 & 0 \\
0 & 0 \\
1 / \mathrm{L}_{\text {ind }} & 0 \\
0 & 1 / \mathrm{L}_{\text {ind }}
\end{array}\right],
\end{gathered}
$$

where $R_{1}=1 \Omega, R_{1}=R_{2}, C_{\text {cap }}=0.1 \mu \mathrm{F}$ and $L_{\text {ind }}=1 H$.

The following matrices $A$ and $B$ represent the dynamics of a fourth-order RLC electrical circuit with two inputs (the voltages from two sources) and two outputs (the voltages of two capacitors). The state vector is defined as $x=\left[\begin{array}{llll}x_{1} & x_{2} & x_{3} & x_{4}\end{array}\right]^{T}$, where $x_{1}$ and $x_{2}$ represent the capacitor voltages, and $x_{3}$ and $x_{4}$ are the currents passing through the inductors. The purpose of the control is to control capacitor voltages and conductor currents in the face of variations in power supplies.

In accordance with the EA-ANN-Core algorithm, the iterative process parameters and initial conditions necessary to obtain the desired eigenstructure are defined as follows:
The desired eigenstructure of (6) and (7) is given by

$$
\begin{aligned}
V_{d}= & {\left[\begin{array}{cccc}
0.4962 & 0.5012 & -0.5038 & 0.4987 \\
-0.4962 & 0.5012 & 0.5038 & 0.4987 \\
-0.5038 & -0.4987 & -0.4962 & 0.5012 \\
-0.5038 & 0.4987 & -0.4962 & 0.5012
\end{array}\right], } \\
\Lambda_{d} & =\operatorname{diag}\left[\begin{array}{llll}
-3.2859 & -4.4819 & -8.4165 & -8.1285
\end{array}\right] .
\end{aligned}
$$

In addition, the ANN auxiliary variables, which are $\bar{a}$, the training rate, and the initial network error, $W_{a n n}(0)$ should be initialized. Next, in line 7 of the algorithm, the iterative process begins, where the algorithm will execute until the number $n$ of eigenvalues of the system, in line 10 is calculated the parameter $\bar{a}_{i}$ that depends on the first pair of the specified structure. In line 12 , the second iterative process begins. After that, the synaptic weights of the network, the error and the gradient are calculated, and then the synaptic weight of the neural network is updated until the error is minimized, thus obtaining $W_{a n n}$, which is the representation of the desired eigenvector with respect to $K$.

Therefore, the values of the resulting weights are:

$$
W_{\text {ann }}=\left[\begin{array}{rrrr}
-0.7036 & 0.1831 & 0.2271 & -0.0043 \\
0.7111 & 0.2739 & -0.2309 & 0.2003
\end{array}\right]
$$

Fig. 3 shows the behavior of the temporal evolution of the synaptic weight $W_{a n n}=\left[\begin{array}{llll}w_{1} & w_{2} & w_{3} & w_{4}\end{array}\right]$ and its convergence. It can be observed that with only 6 iterations there was the convergence of the synaptic weight.
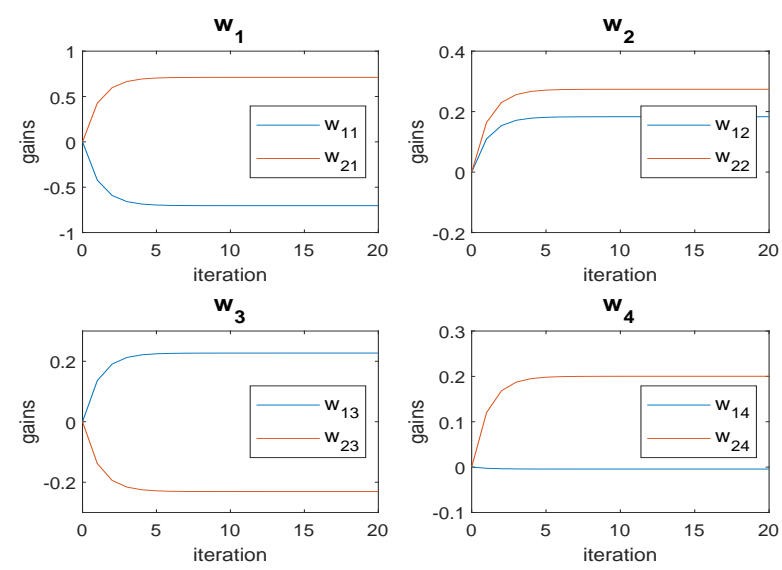

Figure 3. Evolution of the iterative process of the matrix columns $W_{\text {rna }}$ with learning rate $\mu=0.6$.

After completing this step the neural network responsible for obtaining the feedback gain $K_{a n n}$ is initialized online. The auxiliary ANN variables, which are $\bar{B}$, the training rate, the initial network error, and the gain $K_{a n n}(0)$, are initialized.

In sequence, in line 25 of the algorithm an iterative process is started. In line 27 , the parameter $b_{K i}$ is stored, so are the columns of the matrix $W_{a n n}$ at each iteration. The iterative process responsible for getting the $K_{a n n}$ gain starts at line 28, where $N_{\text {imputs }}$ is dependent on the number of system inputs. In line 31 , the second iterative process begins. Next, the synaptic weights of the network, the error, the gradient and then the synaptic weight of the neural network is updated until the error is minimized, thus obtaining $K_{a n n}$. 


$$
K_{\text {ann }}=\left[\begin{array}{rrrr}
0.0394 & 0.0495 & 1.1338 & -0.4913 \\
0.0384 & -0.0560 & -0.6357 & 1.1757
\end{array}\right]
$$

For the purpose of comparison, the calculation of the feedback gain $K_{L S}$ was performed by the classical method of least squares, presented in Section 2.2, the same is presented below

$$
K_{L S}=\left[\begin{array}{rrrr}
0.0379 & 0.0501 & 1.1371 & -0.4894 \\
0.0384 & -0.0560 & -0.6358 & 1.1757
\end{array}\right]
$$

The behavior of the time evolution of the gain $K_{a n n}=\left[k_{1} k_{2}\right]^{T}$ and its convergence are presented in Fig. 4 , i.e. the gains for the first and second control inputs. The learning rate $(\mu)$ used was 0.6 .

From Fig. 4, one notices the convergence of the gains $K_{a n n}$ for the control inputs and it was reached in the 100th iteration.
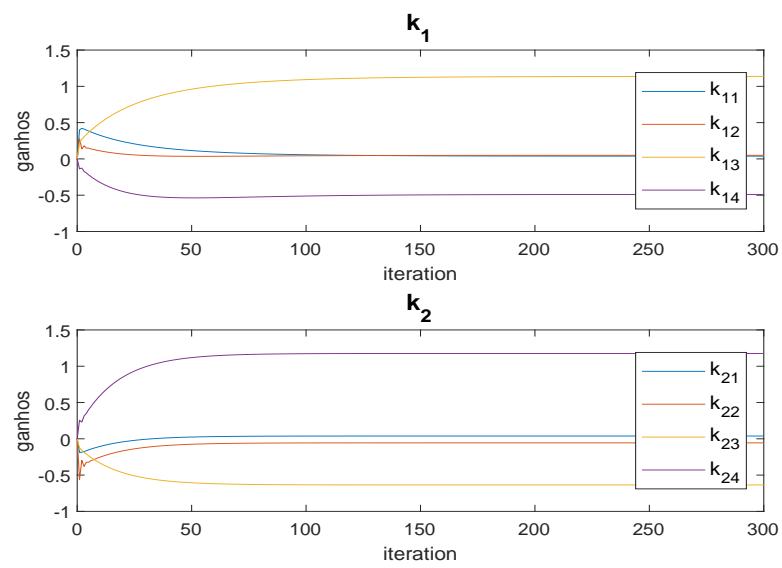

Figure 4. Evolution of the iterative process of the matrix columns $K_{r n a}$ with learning rate $\mu=0.6$.

As can be observed, the neural network proved to be efficient for the total assignment of the desired eigenstructure.

\section{CONCLUSIONS}

The ANN-type Bio-inspired algorithm for the online design of control systems that are dedicated to complete eigenstructure assignment by state feedback was presented in this article. The main objective obtained by this work was to assign online the desired eigenstructure through neural networks. Two neural networks were proposed to solve the problem of EA in MIMO control systems. The first network is responsible for obtaining the representation matrix $W$, and the second network calculates the gain feedback matrix $K$ that assigns the desired eigenstructure. The results are evaluated in mathematical model of an RLC electric circuit.

\section{ACKNOWLEDGMENT}

The authors are indebted to the Federal University of Maranhão (UFMA), the Graduate Program in Electrical Engineering (PPGEE), the State University of Maranhão (UEMA), the Embedded Systems and Intelligent Control Laboratory (LABSECI), the Coordination of Undergraduate Personnel Improvement (CAPES) for the development infrastructure and financial support.

\section{REFERENCES}

Albostan, O. and Gökasan, M. (2018). Mode decoupling robust eigenstructure assignment applied to the lateral-directional dynamics of the f-16 aircraft. Aerospace Science and Technology, 77, $677-687$.

Gajski, D., Abdi, S., Gerstlauer, A., and Schirner, G. (2009). Embedded System Design: Modeling, Synthesis and Verification. Springer US.

Gan, C. and Danai, K. (1999). Fault diagnosis of the ifac benchmark problem with a model-based recurrent neural network. In Proceedings of the 1999 IEEE International Conference on Control Applications, volume 2, 1755-1760 vol. 2.

Haykin, S. (1999). Neural Networks: A Comprehensive Foundation. International edition. Prentice Hall.

Konara, A.I. and Annakkage, U.D. (2016). Robust power system stabilizer design using eigenstructure assignment. IEEE Transactions on Power Systems, 31(3), 1845-1853.

Lee, E. and Seshia, S. (2016). Introduction to Embedded Systems: A Cyber-Physical Systems Approach. Electrical Engineering Computer Sciences. MIT Press.

Liu, D. and Lu, Z. (1997). A new synthesis approach for feedback neural networks based on the perceptron training algorithm. IEEE Transactions on Neural Networks, 8(6), 1468-1482.

Liu, G. and Patton, R. (1998). Eigenstructure assignment for control system design. J. Wiley.

Lu, J., Chiang, H.D., and Thorp, J.S. (1993). Eigenstructure assignment by decentralized feedback control. IEEE Transactions on Automatic Control, 38(4), 587-594.

Sobel, K.M. and Lallman, F.J. (1988). Eigenstructure assignment for the control of highly augmented aircraft. In 1988 American Control Conference, 1266-1276.

Sobel, K.M., y. Shapiro, E., and n. Andry Jr, A. (1994). Eigenstructure assignment. International Journal of Control, 59(1), 13-37.

Wang, L.X. and Wan, F. (2001). Structured neural networks for constrained model predictive control. Automatica, 37(8), $1235-1243$. 